\title{
Gases de efecto invernadero emitidos por los vehículos agrícolas en Costa
} Rica

\author{
Natalia Gómez Calderón ${ }^{1}$, Javier E. Rodríguez Yánez $^{2}$ \& Edwin Fabián Fernández Fernández $^{3}$ (D)
}

1. Instituto Tecnológico de Costa Rica, Escuela de Ingeniería Agrícola, Cartago, CP 159-7050, Costa Rica; ngomez@itcr.ac.cr

2. Universidad Estatal a Distancia, Laboratorio de Ecología Urbana, San José, CP 474-2050, Costa Rica; jrodriguezy@uned.ac.cr

3. Investigador independiente, San José, Costa Rica; ed_f3@hotmail.com

Recibido 11-XI-2020 • Corregido 16-II-2021 • Aceptado 15-IV-2021

DOI: https://doi.org/10.22458/urj.v13i1.3278

\begin{abstract}
Greenhouse gases emitted by agricultural vehicles in Costa Rica". Introduction: In Costa Rica, greenhouse gas emissions from agricultural vehicles are not usually measured independently in inventories. They are usually pooled within overall vehicle fleet or other emissions. Objective: Determine the emissions and specific characteristics for agricultural vehicles in Costa Rica. Methods: The basic characteristics of agricultural machinery in Costa Rica were obtained from information on the payment of circulation taxes for the year 2016. The estimate of emissions was based on the parameters established by the Intergovernmental Panel on Climate Change and factors specific to Costa Rica. Results: Most agricultural machinery is less than 10 years old (92\%), uses diesel fuel (99\%) and is concentrated in the medium power range (category II ASAE S482, 82\%). The total emissions of the equipment under study are 467650 tons of $\mathrm{CO}_{2}$ equivalent, mainly associated with $\mathrm{CO}_{2}$ emissions $(98 \%)$. This is equivalent to $10 \%$ of emissions in the Transport sector and up to $6 \%$ in the Greenhouse Gas Inventory (GHGI). Conclusions: In Costa Rica, emissions from agricultural vehicles are not properly segregated and possibly not properly accounted for. This should be improved in the country's 2050 decarbonization plan.
\end{abstract}

Keywords: Agricultural machinery, emissions, IPCC factors, decarbonization, power engine.
RESUMEN. Introducción: En Costa Rica, la emisión de gases de efecto invernadero de los vehículos agrícolas no suele medirse independientemente en los inventarios. Por lo general, se combinan con los datos del parque general de vehículos $u$ otras emisiones. Objetivo: Determinar las emisiones y características específicas de los vehículos agrícolas en Costa Rica. Métodos: Las características básicas de la maquinaria agrícola en Costa Rica se obtuvieron a partir de información sobre el pago de impuestos a la circulación para el año 2016. La estimación de emisiones se basó en los parámetros establecidos por el Panel Intergubernamental de Cambio Climático y factores propios de Costa Rica. Resultados: La mayoría de la maquinaria agrícola tiene menos de 10 años (92\%), utiliza combustible diésel (99\%) y se concentra en un ámbito medio de potencia (categoría II ASAE S482, $82 \%)$. El total de emisiones de los equipos en estudio es equivalente a 467650 toneladas de $\mathrm{CO}_{2}$, asociado principalmente a emisiones de $\mathrm{CO}_{2}(98 \%)$. Esto es equivalente a un $10 \%$ de las emisiones del sector Transporte y hasta un 6\% en el Inventario de Gases de Efecto Invernadero. Conclusiones: En Costa Rica, las emisiones de los vehículos agrícolas no se segregan adecuadamente y posiblemente no se contabilizan correctamente. Esto debería mejorarse en el plan de descarbonización 2050 del país.

Palabras clave: Maquinaria agrícola, emisiones, factores IPCC, descarbonización, motores de combustión.

El aumento de las emisiones de gases de efecto invernadero (GEI) en la atmósfera es el problema ambiental más urgente en la actualidad (Rebolledo-Leiva, Angulo-Meza, Iriarte, \& González-Araya, 2017). Varias de las actividades asociadas con la agricultura contribuyen a las emisiones de $\mathrm{GEI}$, entre ellas el consumo de energía por parte de la maquinaria agrícola. Dicho parque de maquinaria agrícola es un elemento poco considerado dentro de las evaluaciones de emisiones de gases de efecto invernadero de los parques vehiculares. En Costa Rica, el último 
inventario nacional de gases de efecto invernadero incluye maquinaria agrícola en el sector Transportes, pero sin discriminación, por lo que se desconoce la proporción exacta de emisiones que esta representa (Blanco Salas, Chacón Araya, Jiménez Valverde, Montenegro Vallestero, \& Sasa Marín, 2019).

Por otro lado, esas emisiones dependen mucho del desarrollo de la actividad agrícola; las extensiones de las áreas de cultivo; y sus posibilidades de aplicación en el sitio dependen de la topografía y el motor requerido según las características del suelo que se va a cultivar (Fuglie, 2018). Esto significa que el consumo de combustible fósil utilizado varía en función del tiempo de trabajo de la maquinaria. En Costa Rica, la maquinaria agrícola es subcontratada por tiempo de trabajo (200 horas o un mes, cualquiera que ocurra primero) o área trabajada por hora, según consideraciones comerciales de alquiler y venta de equipos (Gómez-Calderón, Villagra-Mendoza, \& Solórzano, 2018). Los indicadores de consumo de combustible, no se contabilizan o bien las fincas no proveen esa información públicamente. Por lo tanto, no está disponible la cifra exacta del consumo de combustible por la maquinaria agrícola.

Adicionalmente, mejorar la productividad agrícola es fundamental. La Organización para la Cooperación y el Desarrollo Económico, indica que el sector agrícola en Costa Rica debería estar produciendo un 20\% más para el año 2022 de lo que se produjo en el 2018 (Organización para la Cooperación y el Desarrollo Económicos [OCDE], 2018). Los seis cultivos con mayor extensión e importancia económica en Costa Rica son café, pastos, hortalizas, piña, banano y palma africana (Instituto Nacional de Estadísticas y Censos [INEC], 2014), y cubren una superficie cercana al 30\% del país. A excepción de los pastos y el café, los cultivos hacen un uso intensivo de maquinaria agrícola, especialmente cultivos de piña y hortalizas que la utilizan en todos sus procesos de campo (Gómez-Calderón, Solórzano-Quintana, \& Villagra-Mendoza, 2017)

Lo anterior, es un ejemplo de que el proceso de modernización de la agricultura en Costa Rica ha sido más acelerado que en otros países de la región, lo que incluye un aumento significativo en el número de tractores en uso. La tasa de tractores en uso por hectárea de cultivo y cultivo intensivo ha aumentado desde 1961; cuando fue de 0,01 tractores ha ${ }^{-1}$ (Group World Bank, 2016) hasta 1998 donde fue de 13,9 tractores ha-1 (Mora-Alfaro, 2005). Sin embargo, estos vehículos generalmente operan fuera de las rutas de circulación general, por lo que no es posible ver claramente la cantidad de emisiones que estos producen.

Para contabilizar el número de vehículos se considera la existencia de mandatos y registro específicos de maquinaria agrícola para el ingreso y posterior venta en el país. Esto debido a que no existen fabricantes de vehículos agrícolas en Costa Rica. Estos registros representan una base de información confiable basado en las características de la maquinaria agrícola utilizada en el país, de acuerdo con la información proporcionada por el Instituto Nacional de Seguros (INS).

El objetivo de este estudio fue estimar las emisiones de gases de efecto invernadero producidas por el uso de maquinaria agrícola utilizada en Costa Rica, con el fin de recomendar mejoras en el manejo de los datos y emisiones asociados a estos vehículos utilizados en la agricultura. El cálculo se realiza utilizando factores del Panel Intergubernamental de Cambio Climático (IPCC) adaptados para Costa Rica (Chacón Araya, Jiménez Valverde, Montenegro Ballestero, Sasa Marín, \& Blanco Salas, 2015), cuantificando el dióxido de carbono, metano y óxido nitroso, siendo avalados por el Instituto Meteorológico Nacional (2015) para ser utilizados en los inventarios de gases de efecto invernadero en Costa Rica. 


\section{MATERIALES Y MÉTODOS}

Selección de la muestra de maquinaria agrícola: La muestra de vehículos estudiados se obtuvo de la base de datos del impuesto de circulación hasta 2016, proporcionada por el Instituto Nacional de Seguros de Costa Rica (INS), de la que fue seleccionada la categoría llamada "Máquina Especial Agrícola". Con base en esta categoría se establecieron las siguientes restricciones para obtener la muestra analizada:

- Desplazamiento del motor (cilindrada) de $800 \mathrm{~cm}^{3}$ o más.

- Máquinas asociadas a las actividades agrícolas, basadas en la clasificación que realiza el INS para el pago de los impuestos de circulación (maquinaria de barbecho, agro cabañas abiertas y cerradas, maquinaria agrícola, caña cargadora simple, tractores de ruedas y orugas, cosechadoras de algodón, arroz, maíz y caña, aspersores agrícolas).

La muestra obtenida fue agrupada de acuerdo con los siguientes parámetros:

- Número de máquinas por año.

- Cantidad de equipos por tipo de combustible.

- Cantidad de equipos por cilindrada.

Estimación del consumo de combustible y emisiones: La relación de consumo de diésel por unidad de potencia (en kW) del motor (a) es de 0,29 a 0,32 litros de diésel (Alvarado, 2012; GómezCalderón, 2016), para lo cual se debe considerar el grado de esfuerzo de la maquinaria (e). En Costa Rica, se ha estimado que el grado de esfuerzo de los tractores agrícolas es del 54\% (Alvarado, 2004; Food and Agriculture Organization of the United Nations [FAO], 2014), por lo que el consumo promedio de combustible por potencia de cada equipo se puede estimar con ecuación (1).

$$
L_{\text {diésel }}=\boldsymbol{a} * \boldsymbol{P} * \boldsymbol{e} * \boldsymbol{h} \quad \text { Ecuación (1) }
$$

Donde;

$L_{\text {diésel: }} L^{*} \mathrm{mes}^{-1}$ (litros de diésel de potencia por mes por cada máquina agrícola)

$a=0,32$ (límite superior de la relación $\mathrm{L}^{*} \mathrm{~h}^{-1 *} \mathrm{~kW}^{-1}$ )

$P=$ potencia del motor en $\mathrm{kW}$

$e=0,54$

$h=200$ hora* mes $^{-1}$

Luego, la ecuación se reduce a

$$
L_{\text {diésel }}=34,56 * P
$$

\section{Ecuación (2)}

Donde;

$L_{\text {diésel: }} L^{*}$ mes $^{-1}$ (litros de diésel de potencia por mes por cada máquina agrícola)

$\mathrm{P}=$ potencia del motor en $\mathrm{kW}$

Los equipos agrícolas especiales están categorizados en el Tabla 1, de acuerdo con la norma ASAE S482 (ASAE S482, 2004). Las emisiones se estimaron a partir de los factores de emisión del IPCC (Tabla 2) utilizados en el Inventario Nacional de Gases de Efecto Invernadero (INGEI) en Costa Rica en el año 2015 (Instituto Meteorológico Nacional, 2015). Se utilizó la ecuación (3) definida en el INGEI para la estimación de emisiones, aplicando dichos factores por Chacón et al (2015). 
TABLA 1

Categorización de los tractores de acuerdo con la norma ASAE S482

\begin{tabular}{cc}
\hline Categoría de la maquinaria agrícola & Potencia del motor (kW) \\
\hline I & $15-35$ \\
II & $30-75$ \\
III & $60-168$ \\
IV & $135-300$ \\
\hline
\end{tabular}

TABLA 2

Factores de emisión de GEI para fuentes móviles según INGEI en 2014

\begin{tabular}{|c|c|c|c|}
\hline Combustible $^{a}$ & $\begin{array}{c}\mathrm{CO}_{2} \\
\left(\mathrm{~kg} \mathrm{CO}_{2} \mathrm{~L}_{\text {combustible }}^{-1}\right)\end{array}$ & $\begin{array}{c}\mathrm{CH}_{4} \\
\left(\mathrm{~g} \mathrm{CH}_{4} \mathrm{~L}_{\text {combustible }}^{-1}\right)\end{array}$ & $\begin{array}{c}\mathrm{N}_{2} \mathrm{O} \\
\left(\mathrm{g} \mathrm{N}_{2} \mathrm{O} \mathrm{L}_{\text {combustible }}{ }^{-1}\right)\end{array}$ \\
\hline \multirow{2}{*}{ Gasolina } & \multirow{2}{*}{2,26} & $1,0770 *$ & $0,1045 *$ \\
\hline & & $0,8162 * *$ & $0,2612 * *$ \\
\hline Diésel & 2,69 & $0,1416 *$ & $0,1416 *$ \\
\hline GLP & 1,61 & 1,5835 & 0,0051 \\
\hline
\end{tabular}

Fuente: (IMN, 2018)

a factores estimados para 10 años de uso.

* sin catalizador

**con catalizador

Ecuación para calcular las emisiones totales de GEl:

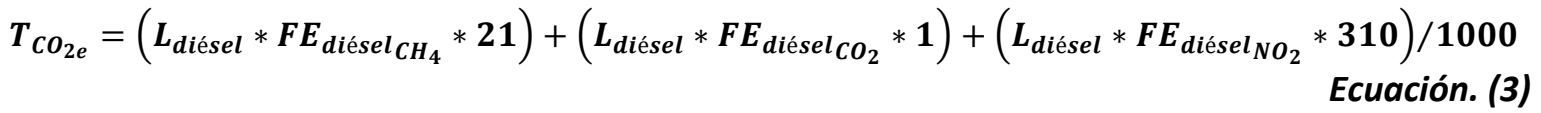

Donde;

$T_{\mathrm{CO}_{2}}$ : Toneladas de dióxido de carbono equivalente.

$L_{\text {diésel }}$ : Litros de diésel

$F E_{\text {diésel }} \mathrm{CH}_{4}$ : Factor de emisiones de metano en la combustión del diésel $\left(\mathrm{kg} \mathrm{CH}_{4}{ }^{*} \mathrm{~L}_{\text {diésel }}{ }^{-1}\right)$ L diésel $^{-1}$ )

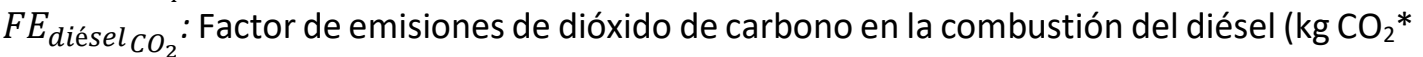

$F E_{\text {diésel }_{\mathrm{NO}_{2}}}$ : Factor de emisiones de óxido nitroso en la combustión del diésel $\left(\mathrm{kg} \mathrm{N}_{2} \mathrm{O}\right.$ * $\mathrm{L}$ diésel ${ }^{-1}$ )

Se aplicó los factores de emisiones de diésel sin un catalizador. En este caso, la ecuación (3) se reduce a la ecuación (4):

$$
T_{C O_{2 e}}=\left(\frac{L_{d i e ́ s e l} * 2,737}{1000}\right) \quad \text { Ecuación (4) }
$$

Donde;

$L_{\text {diésel }}$ : Litros de diésel 
Análisis estadístico: La ecuación (2) se utilizó para obtener los litros totales de diésel utilizados por cada máquina agrícola en un año. Una vez obtenido el volumen de diésel por máquina, se aplicó la ecuación (3) para estimar las emisiones de GEI en 2016 de cada una.

El análisis de la base de datos obtenidos a partir de la estimación con las ecuaciones (2) y (3), se realizó con el software SQL (ISO/IEC 9075 -1:2011, 2011), mientras que los cálculos y estadísticas se realizaron con el software SPSS (IBM, 2016).

\section{RESULTADOS}

El estudio cubrió el 98\% del parque total de maquinaria agrícola en el país hasta 2016, los cuales utilizan primordialmente diésel como fuente de energía. En Costa Rica, el ciclo de vida de la maquinaria agrícola se define en 10000 horas de trabajo, de acuerdo con la Ley 7064 (Ministerio de Agricultura y Ganaderia [MAG], 1984). En el país, se estiman 2500 horas de trabajo anuales en cultivos intensivos, como lo son las plantaciones de piña, mientras que, en cultivos de ciclo corto, como hortalizas, donde solo se realiza labranza del suelo, la jornada laboral se encuentra en un rango entre 500 y 1000 horas anuales. Por este motivo, se consideró maquinaria agrícola de 20 años de antigüedad al año 2016, utilizando un tiempo laboral base 200 horas promedio mensual para los cálculos de consumo promedio de combustible. La asociación entre la cantidad de maquinaria agrícola adquirida por año se muestra en la Fig. 1, así como sus cantidades por tipo de cilindrada (Fig. 2) y combustible (Diesel: 5 740, gasolina: 17, desconocido: 6, alcohol: 1, eléctrico: 1 y Gasolina- GLP: 1).

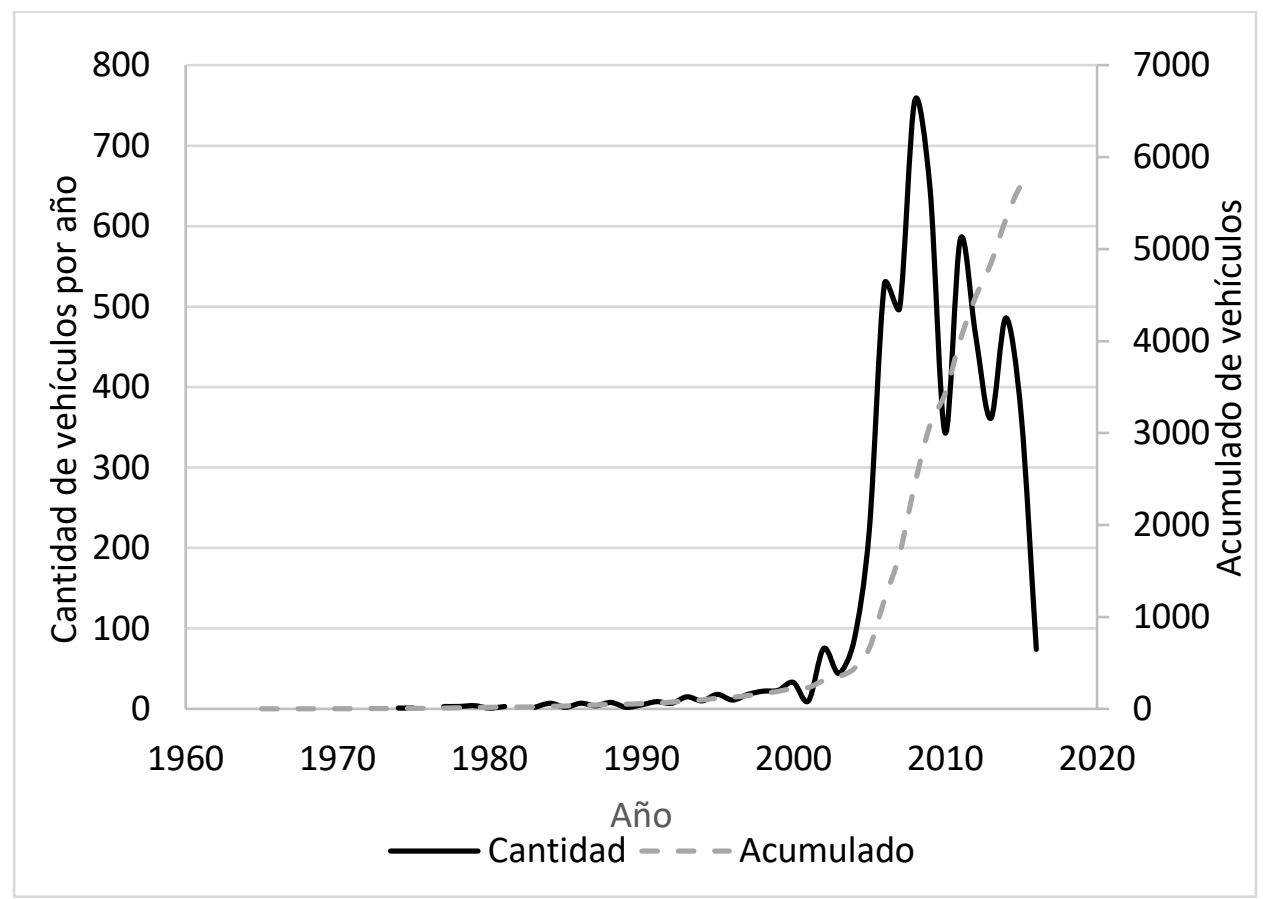

Fig. 1. Número de vehículos de maquinaria agrícola por año y su acumulado. 
La muestra utilizada (5 766 unidades), corresponde en su mayoría a maquinaria agrícola alimentada con diésel como combustible. La mayoría tiene menos de 20 años de uso (Fig. 1), dándose un fuerte incremento desde 2005 al 2016 (92\%), a pesar de que el mercado de vehículos agrícolas se incrementó desde 1998 con el aumento de área del cultivo de piña en el país. Hoy la cantidad de vehículos agrícolas se considera cercana a las necesidades de las áreas de cultivo, con los actuales procesos de compra tendientes a la renovación. Los vehículos agrícolas se compran preferiblemente por marca y pensando en largos periodos de uso, normalmente entre 10 y 20 años (Group World Bank, 2016).

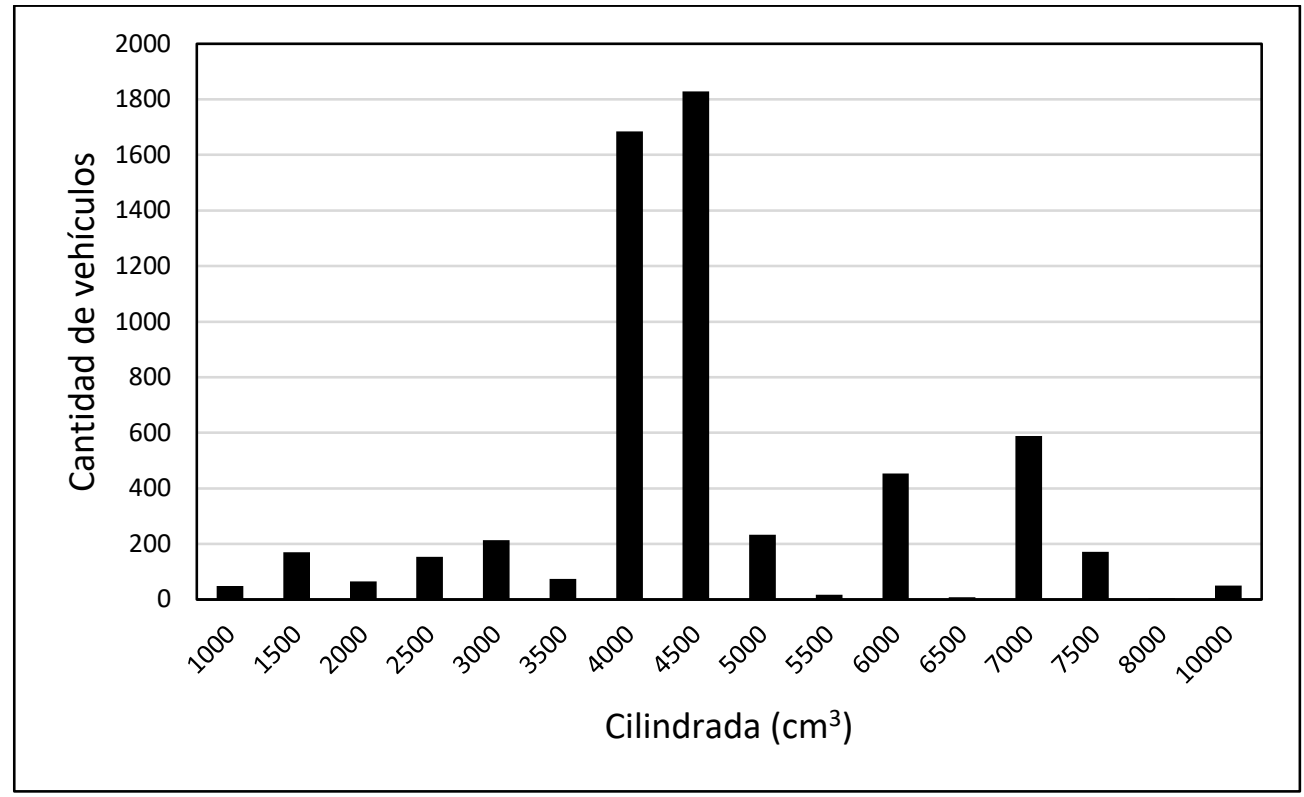

Fig. 2. Número de vehículos de maquinaria agrícola por rango de cilindrada del motor.

La mayor cantidad de vehículos agrícolas utilizados en el país son tractores de categoría II con cilindradas entre 4000 y $4500 \mathrm{~cm}^{3}$ (61\%), comúnmente usados para transportar productos, plantar, cosechar y dar mantenimiento de los principales cultivos del país. La estimación de los GEI expresada en $T_{\mathrm{CO}_{2 e}}$ (Tabla 3) plantea un valor global en el 2016 es de $467648,30 T_{\mathrm{CO}_{2 e}}$, asociado principalmente a emisiones de $\mathrm{CO}_{2}(98 \%)$, para el $95,8 \%$ de la muestra de maquinaria de este estudio. Como es de esperar, las tasas de emisiones de $T_{\mathrm{CO}_{2 e}}$ por vehículo se incrementen con la categoría ASAE S482 del mismo (Tabla 3). Mientras que la mayor proporción de emisiones está relacionada con las categorías de mayor cantidad de vehículos (categoría II y III), representando estas más del $95 \%$ de las emisiones, con cerca del $95 \%$ de los vehículos. La categoría II es la más importante, con el $82,5 \%$ de la flota y el $77,4 \%$ de las emisiones. 
TABLA 3

Estimaciones de las emisiones de GEI por categoría para vehículos agrícolas

\begin{tabular}{|c|c|c|c|c|c|c|c|c|}
\hline Categoría* & Cantidad & $\begin{array}{c}\% \text { de la } \\
\text { flota }\end{array}$ & $\begin{array}{l}\mathrm{CO}_{2} \text { anual } \\
\text { (Ton) }\end{array}$ & $\begin{array}{c}\mathrm{CH}_{4} \text { anual } \\
\left(\mathrm{T}_{\mathrm{CO}_{2 e}}\right)\end{array}$ & $\begin{array}{c}\mathrm{N}_{2} \mathrm{O} \text { anual } \\
\left(\mathrm{T}_{\left.\mathrm{CO}_{2 e}\right)}\right)\end{array}$ & $\begin{array}{c}\text { Total } \\
\left(T_{\left.C_{2 e}\right)}\right)\end{array}$ & $\% \mathrm{CO}_{2} \mathrm{e}$ & $\begin{array}{c}\text { Emisiones } \\
\text { anuales por } \\
\text { unidad } \\
\left(T_{\mathrm{CO}_{2 e}}\right)\end{array}$ \\
\hline I & 233 & 4,22 & 5 199,06 & 5,748 & 84,847 & 5289,65 & 1,13 & 22,7 \\
\hline II & 4555 & 82,50 & $\begin{array}{c}355 \\
948,71\end{array}$ & 393,475 & 5808,439 & 362 150,62 & 77,44 & 79,5 \\
\hline III & 672 & 12,17 & 84838,77 & 93,784 & 1384,429 & 86316,98 & 18,46 & 128,4 \\
\hline IV & 61 & 1,10 & 13653,15 & 15,093 & 222,797 & 13891,04 & 2,97 & 227,7 \\
\hline Total & 5521 & 100 & $\begin{array}{c}459 \\
639,69\end{array}$ & 508,099 & 7500,512 & 467648,30 & & \\
\hline \multicolumn{2}{|c|}{$\begin{array}{l}\text { \% de emisiones } \\
\text { de } \mathrm{CO}_{2 \mathrm{e}}\end{array}$} & & $98,29 \%$ & $0,11 \%$ & $1,60 \%$ & & & \\
\hline
\end{tabular}

*De acuerdo con ASAE S482

\section{DISCUSIÓN}

La mayoría de los vehículos de uso agrícola tienen menos de 10 años, lo cual coincide con la atracción y estimulación de grupos de productores e inversionistas nacionales, frente a nuevas condiciones económicas. Estos lograron aprovechar las oportunidades para la exportación de productos agrícolas no tradicionales (como la piña) y agroindustriales fomentadas desde inicios del siglo XXI (Mora-Alfaro, 2005).

La maquinaria agrícola asociada a estos procesos fueron vehículos para la mecanización de la tierra y el trabajo de carga asociado, relacionadas con el desarrollo de áreas agrícolas pequeñas y medianas (Instituto Nacional de Estadísticas y Censos [INEC], 2014; Gómez-Calderón, N.; SolórzanoQuintana, M.; Villagra-Mendoza, K., 2017). En este sentido, la selección de vehículos con potencias medias permite una utilización continua de los mismos en distintos cultivos, facilitando su repago y mantenimiento. Dentro de estos, se determinó que los vehículos agrícolas son principalmente tractores diésel, con cilindradas entre $4000-5000 \mathrm{~cm}^{3}$ (61\% de la totalidad del parque), asociados a la categoría II (según ASAE S482). Secundariamente, existe otra cantidad importante de vehículos con cilindrada entre los 6000 y $7000 \mathrm{~cm}^{3}$ (18\% del parque), coincidiendo con los tractores de categoría III (según ASAE S482). Estos últimos vehículos son utilizados en labranza de suelos en áreas extensas de topografía menos irregular (Gómez-Calderón, N.; Villagra-Mendoza, K.; Solórz, M., 2018; Gómez-Calderón, 2016).

Las emisiones de $\mathrm{GEl}$ están dominadas por el $\mathrm{CO}_{2}$, representando más de $98 \%$ del total, lo que se asocia tanto al tipo de combustible como al tipo de maquinaria encontrada (tractores con motor de combustión interna de diésel, categoría II y III). Así mismo, las emisiones por unidad incrementan con el aumento de la categoría, asociado principalmente a mayores cilindradas y al consumo de combustible requerido. La cantidad de vehículos de la categoría II (82\%), hace que se genere más de un $77 \%$ de las emisiones. Esta cantidad de emisión sugiere la necesidad de mejorar especialmente la eficiencia en dichos vehículos agrícolas, dado que son equipos ampliamente utilizados en todos los cultivos y todas las operaciones. Para ello, deben ser considerados criterios de tiempo de uso y movimiento para diferentes prácticas agrícolas y sistemas de producción, haciendo planes de gestión de tráfico y uso agrícola controlado.

Los inventarios nacionales no plantean de forma clara la segregación de los vehículos agrícolas (Chacon Araya, Jiménez Valverde, Montenegro Ballestero, Sasa Marín, \& Blanco Salas, 2014; Chacón Araya, Jiménez Valverde, Montenegro Ballestero, Sasa Marín, \& Blanco Salas, 2015; 
Blanco Salas, Chacón Araya, Jiménez Valverde, Montenegro Vallestero, \& Sasa Marín, 2019). La contabilización del sector agrícola se separa en fuentes fijas y fuentes móviles. Las primeras, indicadas en "Otros" sectores como emisiones agrícolas, mientras que las fuentes móviles se contabilizan con el sector "Transporte", sin indicar claramente en que sub-área se encuentra, por lo que se propone que sea considerada en la sub-área de Equipo especial y Otros vehículos. Los valores obtenidos para los INGEI en los años 2010, 2012 y 2015 para estos rubros, son de 330000,370000 y $185000 \mathrm{~T}_{\text {coze }}$ respectivamente. El valor obtenido de emisión en este estudio ( $467648 \mathrm{~T}_{\text {coze }}$ ) resulta muy superior a lo expresado por esas sub áreas en los inventarios. Esto podría indicar que la maquinaria agrícola al no circular por carretera no está siendo considerada específicamente como un grupo de aporte importante de gases de efecto invernadero. El aporte de los vehículos agrícolas podría estar generando un aumento de los valores finales del inventario en el orden de hasta el $10 \%$ para el sector Transporte y del 6\% en el total de emisiones del INGEl.

Adicionalmente Costa Rica a pesar de tener un plan maestro para la descarbonización de la economía para 2050, no considera en forma especial la influencia de los vehículos agrícolas en las emisiones de GEl, por lo que no existe ninguna reglamentación sobre el control de sus emisiones. En este contexto, se considera que el nivel de emisiones indicado en los INGEI para Costa Rica debe ser revisado teniendo en consideración las emisiones de vehículos agrícolas móviles. Aun así, la tendencia actual del aumento de emisiones es cada vez menor, por lo cual los niveles globales no cambiarán sustancialmente en los próximos años (Dirección de Cambio Climático , 2019).

\section{AGRADECIMIENTOS}

Los autores damos un agradecimiento especial al Instituto Nacional de Seguros de Costa Rica, por la contribución de la detallada base de datos, sin la cual este estudio no hubiera sido posible.

\section{ÉTICA, CONFLICTO DE INTERESES Y DECLARACIÓN DE FINANCIAMIENTO}

Los autores declaran haber cumplido con todos los requisitos éticos y legales pertinentes, tanto durante el estudio como en el manuscrito; que no hay conflictos de interés de ningún tipo, y que todas las fuentes financieras se detallan plena y claramente en la sección de agradecimientos. Asimismo, están de acuerdo con la versión editada final del documento. El respectivo documento legal firmado se encuentra en los archivos de la revista.

La declaración de las contribuciones de cada autor al manuscrito son las siguientes: J. R. Y. concepto, obtención de información, métodos, discusión, revisión del documento; N.G. C. manejo de datos, métodos y discusión, revisión del documento; F. F. F. análisis estadístico de datos. 


\section{REFERENCIAS}

Alvarado, A. (2004). Maquinaria y Mecanizacion Agrícola. San José, Costa Rica: EUNED.

Alvarado , A. (2012). Mecanización Agrícola, ¿¿Deterioro o conservación del suelo? Tecnología en Marcha, 19 (1) 56-60.

ASAE S482: 2004. (2004). Drawbars - Agricultural Wheel. Chicago, USA: American Society Agricultural and Biologic - SAI Global Standards.

Blanco Salas, K., Chacón Araya, A., Jiménez Valverde, G., Montenegro Vallestero, J., \& Sasa Marín, J. (2019). Inventario nacional de emisiones por fuente y absorción por sumidero de gases efecto invernadero en Costa Rica 2015. San José, Costa Rica: Instituto Meteorológico Nacional.

Chacón Araya, A., Jiménez Valverde, G., Montenegro Ballestero, J., Sasa Marín, J., \& Blanco Salas, K. (2015). Inventario nacional de gases efecto Invernadero y absorción de carbono, Costa Rica. San José, Costa Rica: Instituto Meteorológico Nacional.

Chacon Araya, A., Jiménez Valverde, G., Montenegro Ballestero, J., Sasa Marín, J., \& Blanco Salas, K. (2014). Inventario nacional de gases efecto invernadero y absorción de carbono 2010. San José, Costa Rica: Instituto Meteorologico Nacional.

Dirección de Cambio Climático . (2019). Plan Nacional de Descarbonizacion 2018-2050. Recuperado de https://cambioclimatico.go.cr/wp-content/uploads/2019/02/PLAN.pdf

Food and Agriculture Organization of the United Nations (FAO). (2014). Estrategia de Mecanización Agrícola. Recuperado de http://www.fao.org/ag/ags/mecanizacion-agricola/estrategia-de-mecanizacion-agricola-ema/es/

Fuglie, K. (2018). Is agricultural productivity slowing?. Global Food Security, 17, 73-83. Doi: 10.1016/j.gfs.2018.05.001

Gómez-Calderón, N. (2016). Fundamentos de maquinria agrícola. San José, Costa Rica: EUNED.

Gómez-Calderón, N.; Solórzano-Quintana, M.; Villagra-Mendoza, K. (2017). Informes de Proyectos de Investigación Cuantificación de la erosión hídrica en función de diferentes técnicas de mecanización para minimizar la contaminación del agua por sedimentos en la parte alta de la cuenca del Río Reventazón. Recuperado de https://repositoriotec.tec.ac.cr/handle/2238/9164

Gómez-Calderón, N.; Villagra-Mendoza, K.; Solórz, M. (2018). La labranza mecanizada y su impacto en la conservación del suelo. Tecnología en Marcha, 31 (1), 167-177. Doi: 10.18845/tm.v31i1.3506

Group World Bank. (2016). Maquinaria agrícola, tractores por cada 100 kilómetros cuadrados de tierra cultivable. Recuperado de https://datos.bancomundial.org/indicador/AG.LND.TRAC.ZS

IBM. (2016). IBM Support - SPSS Modeler 18.0. Recuperado de https://www.ibm.com/support/pages/spss-modeler-180documentation

Instituto Meteorológico Nacional. (2015). Programa de Cambio Climático - Factores de emisión GEl. Recuperado de http://cglobal.imn.ac.cr/documentos/publicaciones/factoresemision/factoresemision2015/offline/download.p df

Instituto Nacional de Estadísticas y Censos (INEC). (2014). VI Censo Nacional Agropecuario: Características de las Fincas y de las Personas Productoras. Recuperado de https://www.inec.go.cr/documento/cenagro-2014-caracteristicasde-las-fincas-y-de-las-personas-productoras-vi-censo-nacion-0

ISO/IEC 9075 -1:2011. (2011). Information technonology - Database languages - SQL - Part 1: Framework. Ginebra, Suiza: International Organization for Standardizationhttps.

Ministerio de Agricultura y Ganaderia (MAG). (1984). Ley 7064 de Fomento a la Producción Agropecuaria. Recuperado de http://www.mag.go.cr/legislacion/1987/Ley-7064.pdf 
Mora-Alfaro, J. (2005). Agrarian policies and rural development in Costa Rica: definitions elements in the new international context. . Agronomía Costarricense, 29 (1), 101-132. Recuperado de https://mpra.ub.uni-muenchen.de/1525/

Organización para la Cooperación y el Desarrollo Económicos (OCDE). (2018). Costa Rica 2018. Estudios Economicos de la OCDE. Recuperado de https://www.oecd.org/eco/surveys/Costa-Rica-2018-Estudios-Economicos-de-laOCDE.pdf

Rebolledo-Leiva, R., Angulo-Meza, L., Iriarte, A., \& González-Araya, M. (2017). Joint carbon footprint assessment and data envelopment analysis for the reduction of greenhouse gas emissions in agriculture production. Science of The Total Environment, ,593-594, 36-46. Doi: 10.1016/j.scitotenv.2017.03.147 